\title{
Risk factors of ineffective drainage in uncovered self-expandable metal stenting for unresectable malignant hilar biliary strictures
}

\author{
Koji Takahashi ${ }^{1}$, Toshio Tsuyuguchi ${ }^{1}$, Atsushi Saiga ${ }^{2}$, Takuro Horikoshi ${ }^{2}$, Yoshihiko \\ Ooka $^{1}$, Harutoshi Sugiyama ${ }^{1}$, Masato Nakamura ${ }^{1}$, Junichiro Kumagai ${ }^{1}$, Mutsumi \\ Yamato $^{1}$, Yotaro Iino ${ }^{1}$, Ayako Shingyoji ${ }^{1}$, Hiroshi Ohyama ${ }^{1}$, Shin Yasui ${ }^{1}$, Rintaro \\ Mikata ${ }^{1}$, Yuji Sakai ${ }^{1}$ and Naoya Kato ${ }^{1}$ \\ ${ }^{1}$ Department of Gastroenterology, Graduate School of Medicine, Chiba University, Chiba, Japan \\ ${ }^{2}$ Department of Radiology, Graduate School of Medicine, Chiba University, Chiba, Japan \\ Correspondence to: Koji Takahashi, email: koji517@gmail.com \\ Keywords: cholangiopancreatography; drainage; endoscopic retrograde; risk factor; stent \\ Received: April 21, $2018 \quad$ Accepted: May 24, $2018 \quad$ Published: June 15, 2018 \\ Copyright: Takahashi et al. This is an open-access article distributed under the terms of the Creative Commons Attribution License \\ 3.0 (CC BY 3.0), which permits unrestricted use, distribution, and reproduction in any medium, provided the original author and \\ source are credited.
}

\section{ABSTRACT}

Aim: In this study, we assessed the factors contributing to ineffective drainage in the initial transpapillary uncovered self-expandable metal stent (USEMS) placements in patients with unresectable malignant hilar biliary strictures (UMHBSs) (Bismuth type II or higher).

Methods: This was a retrospective, single-center study. A total of 97 patients with UMHBSs who underwent technically successful initial USEMS placements using endoscopic retrograde cholangiopancreatography (ERCP) were classified into the effective drainage group $(n=73)$ or the ineffective drainage group $(n=24)$. We then compared group characteristics, clinical outcomes, and drained liver volumes. Drained liver volume was measured by using computed tomography volumetry. The definition of effective biliary drainage was a $50 \%$ decrease in the serum total bilirubin level or normalization of the level within 14 days of stent placement.

Results: Univariate analysis showed that ineffective drainage was associated with the pre-ERCP serum total bilirubin level $(P=0.0075)$, pre-ERCP serum albumin level $(P=0.042)$, comorbid liver cirrhosis $(P=0.010)$, drained liver volume $(P=$ $0.0010)$, and single stenting $(P=0.022)$. Multivariate analysis identified comorbid liver cirrhosis (adjusted odds ratio [OR], 5.79; 95\% confidence interval [CI], 1.3025.85; $P=0.022$ ) and drained liver volume $<50 \%$ (adjusted $O R, 5.50 ; 95 \% C I$, 1.50-20.25; $P=0.010$ ) as independent risk factors of ineffective drainage.

Conclusion: Comorbid liver cirrhosis and a drained liver volume $<50 \%$ contributed significantly to ineffective drainage in the initial transpapillary USEMS placements for UMHBSs.

\section{INTRODUCTION}

A malignant hilar biliary stricture (MHBS) is caused by cholangiocarcinoma, gallbladder carcinoma, metastatic liver tumors, or hilar lymph node metastases from various cancers. MHBSs induce high serum bilirubin concentrations due to cholestasis (obstructive jaundice). Obstructive jaundice affects the biliary tree, and the hepatic cell and liver functions. The loss of bile in the gut disrupts the intestinal mucosal barrier, which increases the absorption of endotoxin from the intestinal tract. The resulting endotoxemia causes inflammatory cytokinesis and induces a systemic inflammatory response syndrome, which may lead to a multiple organ dysfunction syndrome [1]. 
Effective biliary drainage is essential to improve the quality of life of patients with MHBS. Plastic and metallic stents (MSs) are available for biliary drainage in patients with unresectable MHBSs (UMHBSs), and studies have shown that MSs are superior in terms of their patency period and cost effectiveness $[2,3]$. Thus, uncovered self-expandable MSs (USEMSs) are now mainly used for drainage in patients with UMHBSs. Metal stenting methods are diverse and include side-by-side placement (SBS), partial stent-in-stent placement (PSIS), and single stenting (Figure 1). However, there are no unified guidelines with regard to the drained liver volume and the stenting method involving a MS for a UMHBS [4]. Furthermore, the factors contributing to ineffective drainage in the initial transpapillary USEMS placements in patients with UMHBSs are unclear. In the TOKYO Criteria 2014, functional success, which indicates effective biliary drainage, was defined as a $50 \%$ decrease in the serum total bilirubin level or normalization of the serum total bilirubin level within 14 days of stent placement [5]. This study assessed factors contributing to ineffective drainage in the initial transpapillary USEMS placements in patients with UMHBSs (Bismuth type II or higher).

\section{RESULTS}

A total of 97 patients with UMHBSs who underwent technically successful first-time endoscopic SEMS placement for biliary drainage at the Chiba University Hospital between July 2005 and September 2017 contributed data for this analysis (Figure 2). The patients' characteristics and treatment outcomes are shown in Table 1. There were 67 men and 30 women (median [interquartile range, IQR] age, 69 [63-78] years). Of the 97 patients, 34 underwent SBS, 18 underwent PSIS, and 45 underwent single stenting. For SBS and PSIS, no cases underwent stent placements in three or more branches of the bile duct in a single procedure. All patients had
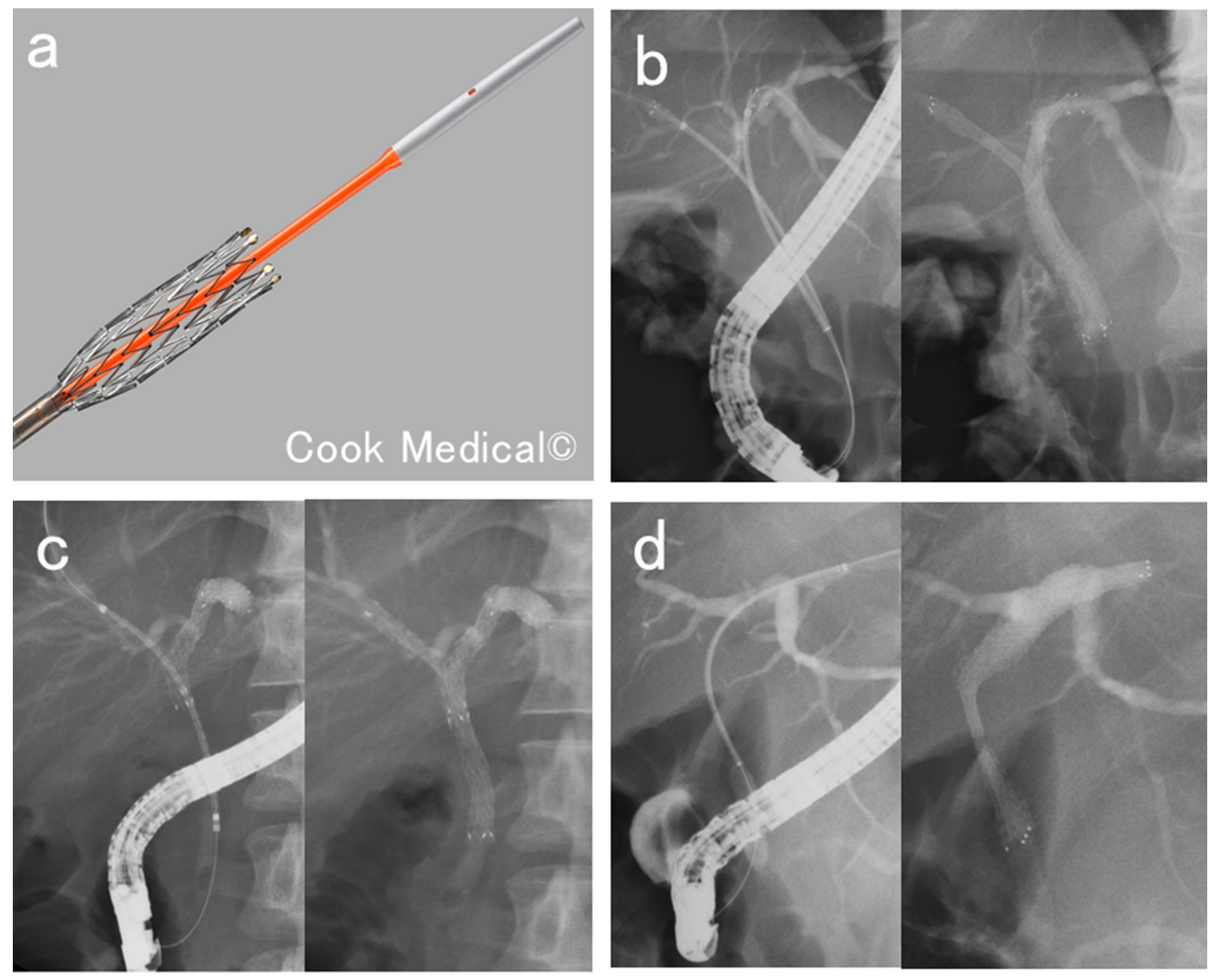

Figure 1: Methods of biliary metal stenting for malignant hilar biliary strictures. (a) Uncovered self-expandable metal stent and its delivery system. (b) Side-by-side placement. Two metal stents inserted parallel to each other into different branches of the hepatic bile duct. (c) Partial stent-in-stent placement. A second metal stent inserted into a different branch of the hepatic bile duct system through the mesh of the first metal stent. (d) Single stenting. Metal stent inserted into one branch of the hepatic bile duct. 
undergone endoscopic sphincterotomy before the SEMS placements. No patients had marked thrombocytopenia, and no patients had dialysis requirements.

Of the 97 eligible patients, data from 73 were included in the effective drainage group, and data from 24 were included in the ineffective drainage group (Table 2). Univariate analysis identified the significant factors contributing to ineffective drainage as the pre- endoscopic retrograde cholangiopancreatography (ERCP) serum total bilirubin level $(P=0.0075)$, pre-ERCP serum albumin level $(P=0.042)$, comorbid liver cirrhosis $(P=0.010)$, drained liver volume $(P=0.0010)$, and single stenting $(P$ $=0.022$ ). The types of indications, methods of previous biliary drainage, and Bismuth classification types were not significant contributing factors.

The areas under the receiver operating characteristic (ROC) curve for the pre-ERCP serum total bilirubin level, pre-ERCP serum albumin level, and drained liver volume were $0.683,0.639$, and 0.724 , respectively. We calculated cutoff values for the pre-ERCP serum total bilirubin level (4.8 mg/dl; sensitivity, 75\%; specificity, 68\%), pre-ERCP serum albumin level $(2.6 \mathrm{mg} / \mathrm{dl}$; sensitivity, $50 \%$; specificity, 81\%), and drained liver volume were (50\%; sensitivity, 75\%; specificity, 74\%) (Figure 3). We classified the pre-ERCP serum total bilirubin level, preERCP serum albumin level, and drained liver volume according to the cutoff values (Table 3), and then performed a multivariate analysis.
The multivariate analysis used the five items that had shown significance in the univariate analysis (Table 4). Our multivariate analysis identified comorbid liver cirrhosis (adjusted odds ratio [OR], 5.79; 95\% confidence interval [CI], 1.30-25.85; $P=0.022$ ) and drained liver volume $<50 \%$ (adjusted OR, 5.50; 95\% CI, 1.50-20.25; $P=0.010)$ as independent risk factors of ineffective drainage.

Complications occurred in 10 patients $(10 \%)$. Five patients had cholangitis, two had pancreatitis, and one each had hepatic abscess, pneumonia, and heart failure. In the patient with a hepatic abscess, the abscess formed in a non-drained area, and additional stenting was required. The other complications in patients were quickly relieved, and no deaths occurred in association with the SEMS placement.

\section{DISCUSSION}

Our study results identified comorbid liver cirrhosis and drained liver volume $<50 \%$ as significant risk factors of ineffective drainage.

Reports have mentioned that single stenting is sufficient for reducing the bilirubin level of patients with MHBSs [6, 7]. A prospective study on 35 patients showed that unilateral metal stenting using magnetic resonance cholangiopancreatography or computed tomography (CT) to selectively target drainage, provided safe and

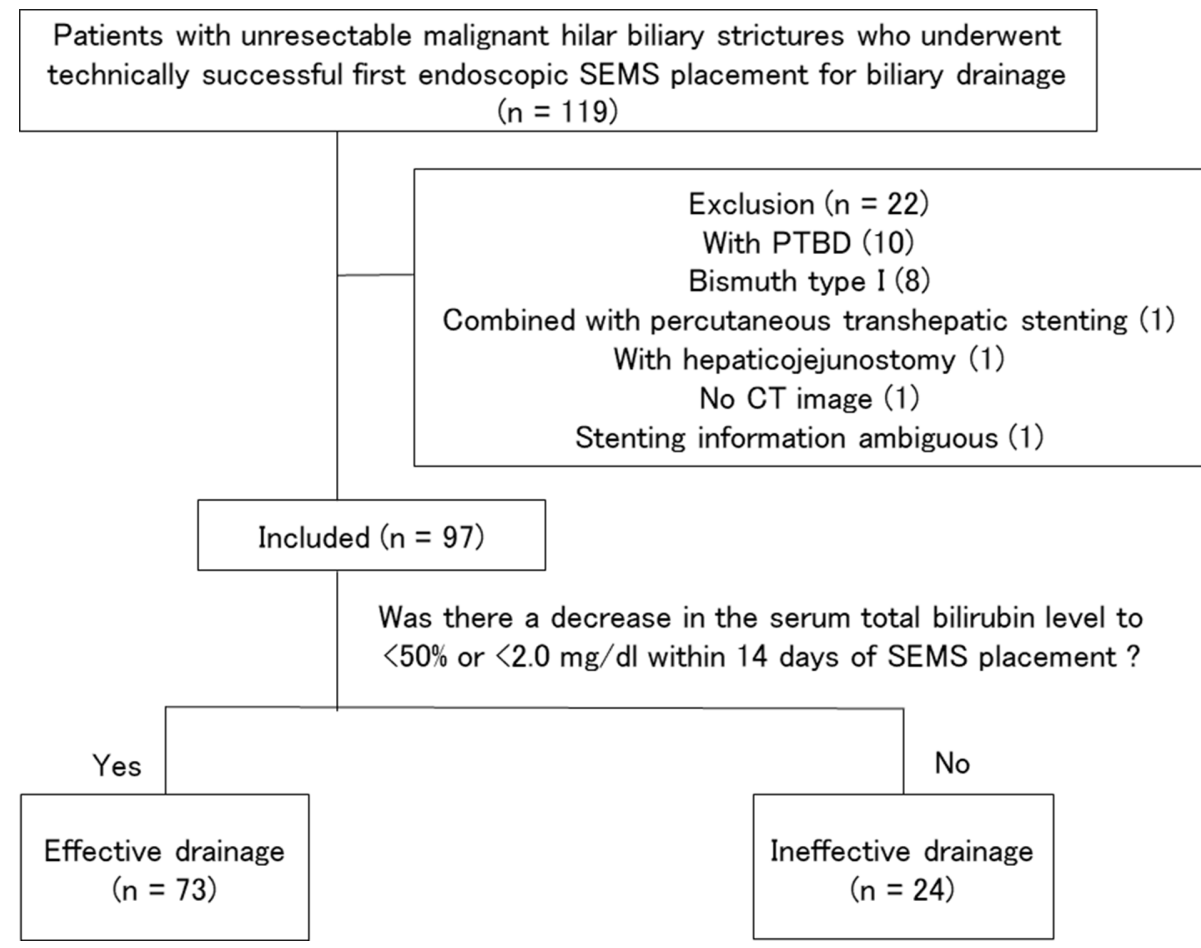

Figure 2: Diagram of case selection flow. 
Table 1: Clinical features of the 97 patients

\section{Characteristics and clinical outcomes}

Age, median (IQR)

Gender, male, n (\%)

Performance status, median (IQR)

Indications, $\mathrm{n}(\%)$

Cholangiocarcinoma

Gallbladder carcinoma

Hepatocellular carcinoma

Metastatic liver tumor

Hilar lymph nodes metastasis

Method of previous biliary drainage, n (\%)

No history of previous biliary drainage

Plastic stent

ENBD

PTBD

Pre-ERCP serum total bilirubin level, mg/dl, median (IQR)

Pre-ERCP serum albumin level, mg/dl, median (IQR)

Comorbid cholangitis, $\mathrm{n}(\%)$

Comorbid liver cirrhosis, n (\%)

Bismuth classification type, n (\%)

Type II

Type III

Type IV

Stenting method, n (\%)

Side-by-side placement

Partial stent-in-stent placement

Single stenting

Stent placement above the papilla, n (\%)

Drained liver volume, \%, median (IQR)

Effective drainage, n (\%)

Complication, n (\%)
69 (63-78)

$67(69 \%)$

$1(1-3)$

$54(56 \%)$

$17(18 \%)$

$14(14 \%)$

$10(10 \%)$

$2(2 \%)$

$23(24 \%)$

37 (38\%)

$36(37 \%)$

$1(1 \%)$

$4.8(1.7-9.8)$

$2.9(2.6-3.3)$

$18(19 \%)$

$16(16 \%)$

$12(12 \%)$

$20(21 \%)$

$65(67 \%)$

$34(35 \%)$

$18(19 \%)$

$45(46 \%)$

$90(93 \%)$

53.5 (42.3-61.8)

$73(75 \%)$

$10(10 \%)$

Abbreviations: IQR, interquartile range; ENBD, endoscopic retrograde biliary drainage; PTBD, percutaneous transhepatic biliary drainage; ERCP, endoscopic retrograde cholangiopancreatography

effective palliation in most patients with MHBSs [6]. Another prospective study on 61 patients showed that unilateral MS placement was safe and feasible and achieved adequate drainage in most patients with unresectable hilar cholangiocarcinoma [7]. In recent years, many studies have reported on the efficacy of multiple stenting [8-13]. However, there are few reports on factors contributing to ineffective drainage, and these factors are not clear. Drained liver volume is considered to be important for effective drainage, and drained liver volume has been assessed using CT volumetry [14, 15]. Vennie et al. reported that drainage $>50 \%$ of the variable liver volume is an important predictor of drainage and signals effective palliation in patients with MHBSs [14]. 
Table 2: Univariate analysis for risk factors of ineffective biliary drainage

\begin{tabular}{|c|c|c|c|}
\hline & Effective drainage & Ineffective drainage & \\
\hline & $\mathrm{n}=\mathbf{7 3}$ & $\mathrm{n}=\mathbf{2 4}$ & $T$-value \\
\hline Age, median (IQR) & $71(64-79)$ & $67(61-71)$ & 0.056 \\
\hline Gender, male, n (\%) & $49(67 \%)$ & $18(75 \%)$ & 0.47 \\
\hline Performance status, median (IQR) & $1(1-3)$ & $1(1-2)$ & 0.27 \\
\hline Cholangiocarcinoma, n (\%) & $44(60 \%)$ & $10(42 \%)$ & 0.11 \\
\hline Gallbladder carcinoma, n (\%) & $12(16 \%)$ & $5(21 \%)$ & 0.62 \\
\hline Hepatocellular carcinoma, n (\%) & $10(14 \%)$ & $4(17 \%)$ & 0.72 \\
\hline Metastatic liver tumor, n (\%) & $6(8 \%)$ & $4(17 \%)$ & 0.24 \\
\hline Hilar lymph nodes metastasis, n (\%) & $1(1 \%)$ & $1(4 \%)$ & 0.40 \\
\hline \multicolumn{4}{|l|}{ Method of previous biliary drainage, n (\%) } \\
\hline No history of previous biliary drainage, $\mathrm{n}(\%)$ & $15(21 \%)$ & $8(33 \%)$ & 0.20 \\
\hline Plastic stent & $31(42 \%)$ & $6(25 \%)$ & 0.13 \\
\hline ENBD & $27(37 \%)$ & $9(38 \%)$ & 0.96 \\
\hline PTBD & 0 & $1(4 \%)$ & 0.080 \\
\hline $\begin{array}{l}\text { Pre-ERCP serum total bilirubin level, mg/dl, } \\
\text { median (IQR) }\end{array}$ & $3.9(1.5-7.7)$ & $8.2(4.6-13.9)$ & 0.0075 \\
\hline $\begin{array}{l}\text { Pre-ERCP serum albumin level, } \mathrm{mg} / \mathrm{dl} \text {, median } \\
\text { (IQR) }\end{array}$ & $2.9(2.7-3.3)$ & $2.7(2.1-3.2)$ & 0.042 \\
\hline Comorbid cholangitis, n (\%) & $11(15 \%)$ & $7(29 \%)$ & 0.12 \\
\hline Comorbid liver cirrhosis, n (\%) & $8(11 \%)$ & $8(33 \%)$ & 0.010 \\
\hline \multicolumn{4}{|l|}{ Bismuth classification type, n (\%) } \\
\hline Type II & $10(14 \%)$ & $2(8 \%)$ & 0.49 \\
\hline Type III & $14(19 \%)$ & $6(25 \%)$ & 0.54 \\
\hline Type IV & $49(67 \%)$ & $16(67 \%)$ & 0.97 \\
\hline Drained liver volume, \%, median (IQR) & $56(49-64)$ & $40(34-51)$ & 0.0010 \\
\hline Side-by-side placement, n (\%) & $29(40 \%)$ & $5(21 \%)$ & 0.092 \\
\hline Partial stent-in-stent placement, n (\%) & $15(21 \%)$ & $3(13 \%)$ & 0.38 \\
\hline Single stenting, $\mathrm{n}(\%)$ & $29(40 \%)$ & $16(67 \%)$ & 0.022 \\
\hline Stent placement above the papilla, n $(\%)$ & $67(92 \%)$ & $23(96 \%)$ & 0.51 \\
\hline
\end{tabular}

Abbreviations: IQR, interquartile range; ENBD, endoscopic retrograde biliary drainage; PTBD, percutaneous transhepatic biliary drainage; ERCP, endoscopic retrograde cholangiopancreatography.

Takahashi et al. reported that a liver volume drainage $\geq$ $33 \%$ in patients with preserved liver function correlates with effective biliary drainage in cases of UMHBSs [15]. Both these studies employed various biliary drainage methods. However, our study focused on only the initial USEMS placements in patients with UMHBSs. To our knowledge, this is the first study to investigate the factors contributing to ineffective drainage after the initial metal stenting for UMHBSs.
Our multivariate analysis showed that comorbid liver cirrhosis and drained liver volume $<50 \%$ were independent significant factors contributing to ineffective drainage. Based on that, a stenting method draining $\geq 50 \%$ of the liver volume should be planned for the first transpapillary USEMS placement in patients with UMHBSs (Bismuth type II or higher). In cases with Bismuth type II or III, this is achievable with single stenting, but in Bismuth type IV cases, multiple stenting 
Table 3: Rate of ineffective drainage when divided into two groups according to the cut-off value

\begin{tabular}{|c|c|c|c|}
\hline & Effective drainage & Ineffective drainage & \multirow{2}{*}{$P$-value } \\
\hline & $\mathbf{n}=\mathbf{7 3}$ & $n=24$ & \\
\hline Pre-ERCP serum total bilirubin level $\geq 4.8 \mathrm{mg} / \mathrm{dl}, \mathrm{n}(\%)$ & $31(42 \%)$ & $18(75 \%)$ & 0.0057 \\
\hline Pre-ERCP serum albumin level $\leq 2.6 \mathrm{mg} / \mathrm{dl}, \mathrm{n}(\%)$ & $15(21 \%)$ & $12(50 \%)$ & 0.0052 \\
\hline Drained liver volume $<50 \%, \mathrm{n}(\%)$ & $19(26 \%)$ & $17(71 \%)$ & 0.0001 \\
\hline
\end{tabular}

Abbreviations: ERCP, endoscopic retrograde cholangiopancreatography

is necessary to drain $\geq 50 \%$ of the liver volume, and multiple stenting should be attempted. It should also be noted that effective drainage is more difficult in patients with liver cirrhosis than in patients with a normal liver. A larger drainage area seems to be required in patients with impaired liver function than in patients with preserved liver function [15]. In this study, we took the presence of liver cirrhosis into consideration, and impaired liver function or preserved liver function was not classified. The required drained liver volume for effective drainage
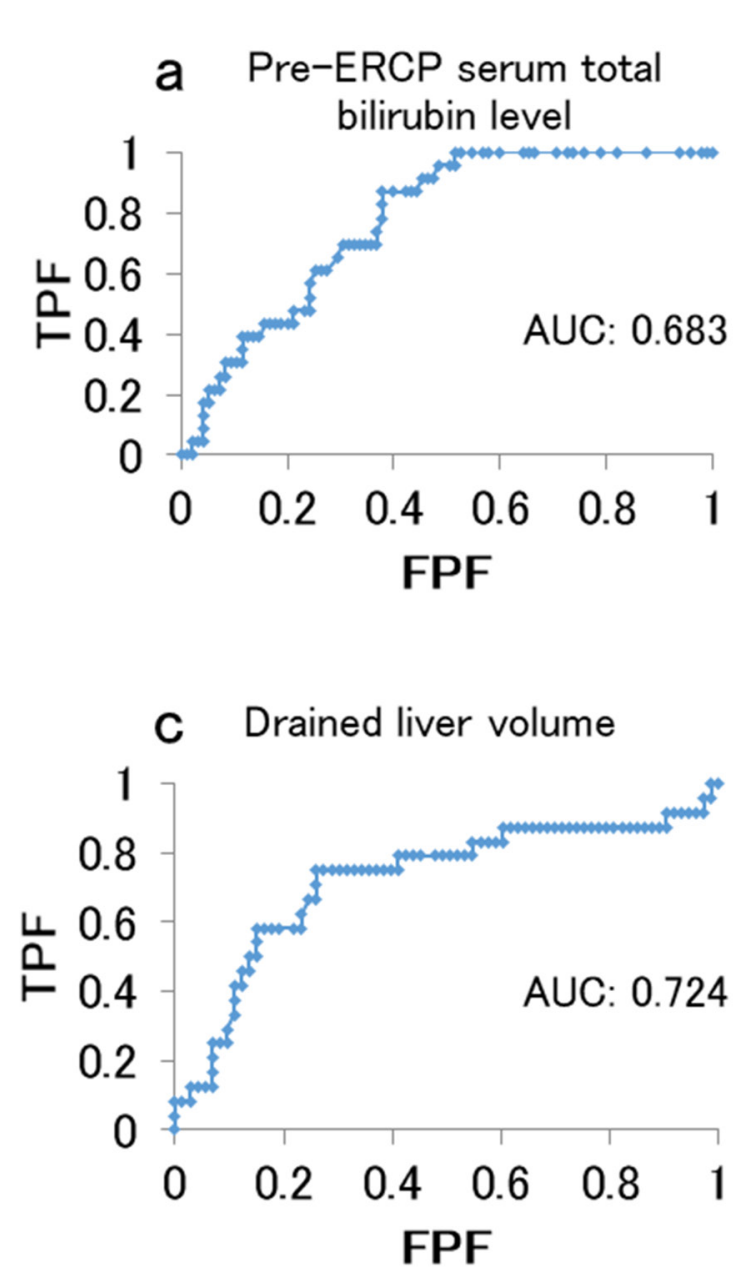

may change in relation to the liver functionality, and for patients with cirrhosis, a drained liver volume $>50 \%$ might be necessary. The number of patients with liver cirrhosis was small in our study, and a larger study is required to assess the conditions necessary for effective drainage in patients with cirrhosis.

We are aware of the limitations in our study. First, this is a retrospective study at a single-center and the number of patients whose data we analyzed was limited to 97. Second, our study population targeting patients with

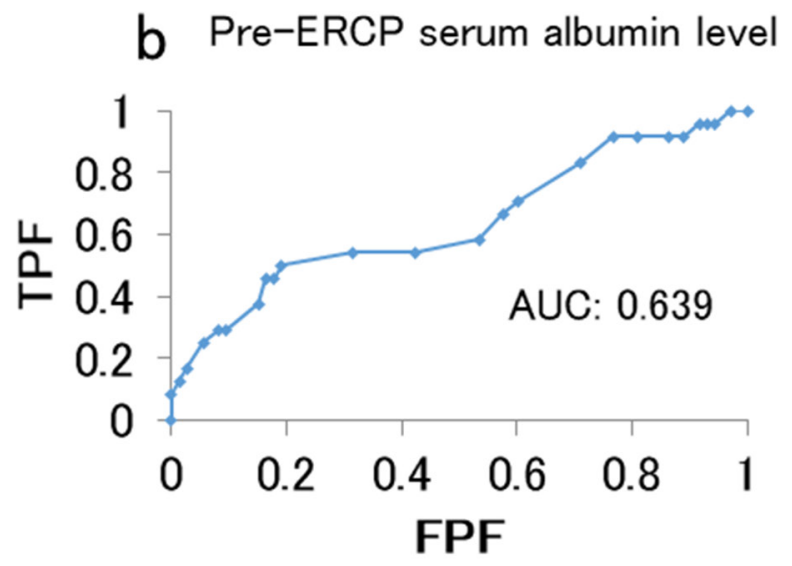

Figure 3: Area under the receiver operating characteristic curve for predicting effective drainage. (a) Pre-stenting serum total bilirubin level. (b) Pre-stenting serum albumin level. (c) Drained liver volume. Abbreviations: FPF, false positive fraction; TPF, true positive fraction. 
Table 4: Multivariate analysis for the risk factors of ineffective biliary drainage

\begin{tabular}{lcccc}
\hline & \multirow{2}{*}{ Adjusted odds ratio } & \multicolumn{2}{c}{$\mathbf{9 5 \%}$ confidence interval } & \multirow{2}{*}{$\boldsymbol{P}$-value } \\
\cline { 3 - 4 } & & Lower & Upper & \\
\hline Pre-ERCP serum total bilirubin level $\geq 4.8 \mathrm{mg} / \mathrm{dl}$ & 3.45 & 0.96 & 12.42 & 0.058 \\
Pre-ERCP serum albumin level $\leq 2.6 \mathrm{mg} / \mathrm{dl}$ & 3.15 & 0.88 & 11.29 & 0.077 \\
Single stenting & 1.42 & 0.36 & 5.55 & 0.620 \\
Comorbid of liver cirrhosis & 5.79 & 1.30 & 25.85 & 0.022 \\
Drained liver volume $<50 \%$ & 5.50 & 1.50 & 20.25 & 0.010 \\
\hline
\end{tabular}

Abbreviations: ERCP, endoscopic retrograde cholangiopancreatography

different types of diseases was heterogeneous. Finally, our CT volumetry may not be the most accurate test for evaluating drained liver volume, particularly in patients with high stenosis, and the assessment method should be improved for future studies.

In conclusion, the significant factors contributing to ineffective drainage after an initial transpapillary
USEMS placement for UMHBSs are comorbid liver cirrhosis and drained liver volume $<50 \%$. The strategy for the first transpapillary USEMS placement in patients with UMHBSs (Bismuth type II or higher) should involve stenting for draining $\geq 50 \%$ of the liver volume to achieve effective drainage. A prospective study is needed to validate the results of our study.

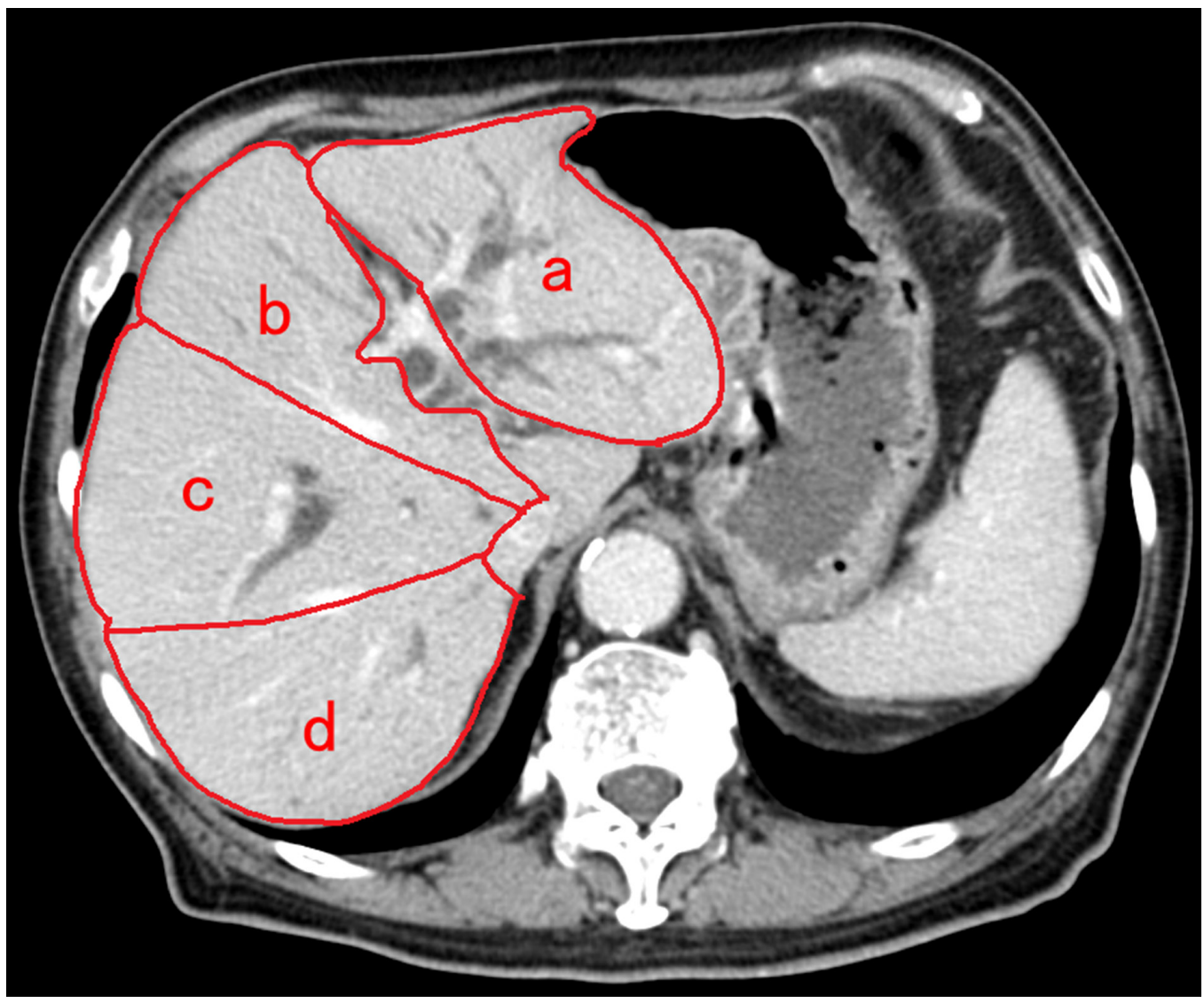

Figure 4: Method of identification of each liver section and computed tomography volumetry. Four sectors of liver were defined based on the distribution of portal vein branches, excluding the caudate lobe. The areas of the left lateral sector (a), left medial sector (b), right anterior sector (c), and right posterior sector (d) were measured in computed tomography (CT) images. Additionally, the area of each sector was measured by tracing CT images, and then summing up the area of the CT slices comprising that sector. 


\section{PATIENTS AND METHODS}

\section{Study design}

This was a retrospective, single-center study. We reviewed the medical records of patients and compared the characteristics and clinical outcomes between those with effective drainage (effective drainage group) and those without effective drainage (ineffective drainage group) after USEMS placement for a UMHBS. Additionally, we calculated the estimated drained liver volume using CT volumetry. Moreover, we compared various factors between the two groups. Significant factors contributing to ineffective drainage were identified in a multivariate analysis. The ethics committee of the Chiba University Hospital approved this study.

\section{Patients}

Patients with UMHBSs who underwent technically successful first-time endoscopic SEMS placement for biliary drainage at the Chiba University Hospital between July 2005 and September 2017 were considered for inclusion. We identified a total of 119 consecutive patients through retrospective analysis of the prospectively recorded endoscopic database in our hospital. Technical success was defined as SEMS placement with sufficient coverage of the bile duct stricture. The exclusion criteria were as follows: percutaneous transhepatic biliary drainage was continued even after metal stenting, Bismuth type I condition, percutaneous transhepatic metal stenting, biliary tract reconstruction, no abdominal CT scan within 14 days before stenting, and ambiguous stenting information.

\section{Techniques}

Transpapillary SEMS placements for biliary drainage were performed via therapeutic duodenoscopy (JF $260 \mathrm{~V}$ or TJF $260 \mathrm{~V}$; Olympus, Tokyo, Japan) using the ERCP technique. The SEMSs included Zilver635 biliary stent (Cook Japan, Tokyo, Japan), X-Suit NIR (Olympus, Tokyo, Japan), WallFlex (Boston Scientific Japan, Tokyo, Japan), Niti-S D-type (Century Medical, Tokyo, Japan), BILERUSH (PIOLAX, Yokohama, Japan), and JOSTENT (Zeon Medical, Tokyo, Japan). In cases of SBS and PSIS, which require the stents to be place into two branches of the bile duct, the stenting was completed with a single ERCP in all relevant cases. In this study, the SBS and PSIS were considered as multiple stenting procedures. On the other hand, stenting in a single branch of the bile duct was considered as single stenting.

\section{Methods}

For this study, patients satisfying the functional success criteria of the TOKYO Criteria 2014 without additional interventions were included in the effective drainage group, and the remaining patients were included in the ineffective drainage group. We defined functional success as a decrease in the serum total bilirubin level to $<50 \%$ or $<2.0 \mathrm{mg} / \mathrm{dl}$ within 14 days of SEMS placement without additional biliary treatments. We compared the clinical characteristics, stenting methods, clinical outcomes, and estimated drained liver volumes between the groups. We analyzed the following variables in the effective and ineffective drainage groups: age, gender, Eastern Cooperative Oncology Group performance status, types of indications, Bismuth types [16], stenting methods, complications, pre-ERCP serum total bilirubin levels, pre-ERCP serum albumin levels, comorbid rates of cholangitis, comorbid rates of liver cirrhosis, and estimated drained liver volumes. The Bismuth types were based on the retrograde cholangiography and CT findings comprehensively. We evaluated the complication events according to the TOKYO Criteria 2014. Liver cirrhosis was judged by two gastroenterologists. Patients with medical history and laboratory data suggestive of cirrhosis and findings characteristic of liver cirrhosis on abdominal ultrasound, CT, or magnetic resonance imaging were considered to have liver cirrhosis.

For measurement of estimated drained liver volume using CT volumetry, we referred to previous reports [14, $15]$ and consultation was performed with the radiological department and gastroenterological department in Chiba University Hospital, and then, the assessment method was decided. According to the distribution of portal vein branches, four sectors of the liver were defined, excluding the caudate lobe. The sectors were the left lateral sector, left medial sector, right anterior sector, and right posterior sector. The areas of these sectors were measured in CT images (Figure 4). We manually traced the area of each sector using axial CT images with a 5-mm slice thickness, including the tumor component, to calculate the volume of each sector (summed area of the slices comprising the sector). Then, we calculated the drained liver volume, which did not include the tumor component, on the basis of the stent position, the non-tumor volume of each liver sector, and the type of bile duct stricture, according to the Bismuth classification. Additionally, we calculated the ratios of the drained liver volume to the total live volume.

With regard to the effectiveness of previous drainage, some cases changed from previous drainage to USEMSs within a few days, and thus, it was difficult to evaluate the effectiveness of previous drainage.

\section{Statistical analysis}

We performed univariate analysis for comparisons between the effective and ineffective drainage groups. Pearson's chi-squared test was used to assess categorized data, while the Mann-Whitney $U$ test was used to assess quantitative data. For multivariate analysis, we performed binomial logistic regression analysis on items that showed 
significance in the univariate analysis. For quantitative data, categorization was performed using cutoff values calculated by determining the smallest distance between the receiver operating characteristic (ROC) curve and the upper left corner of the graph, and multivariate analysis was performed using that categorized data. Data are presented as median (interquartile range $[\mathrm{IQR}])$ or number $(\%)$. A $P$-value $<$ 0.05 was considered statistically significant. All statistical analyses were performed using BellCurve for Excel (Social Survey Research Information Co., Ltd., Tokyo, Japan).

\section{Abbreviations}

USEMS, uncovered self-expandable metal stent; UMHBS, unresectable malignant hilar biliary stricture; ERCP, endoscopic retrograde cholangiopancreatography; MHBS, malignant hilar biliary stricture; MS, metal stent; SBS, side-by-side placement; PSIS, partial stent-in-stent placement; CT, computed tomography; ROC, receiver operating characteristic; IQR, interquartile range.

\section{Author contributions} Kato.

Study supervision: Toshio Tsuyuguchi and Naoya

Endoscopic operation: Koji Takahashi, Toshio Tsuyuguchi, Harutoshi Sugiyama, Masato Nakamura, Junichiro Kumagai, Mutsumi Yamato, Yotaro Iino, Hiroshi Ohyama, Shin Yasui, Rintaro Mikata, and Yuji Sakai.

Image analysis: Koji Takahashi, Toshio Tsuyuguchi, Atsushi Saiga, and Takuro Horikoshi.

Postoperative management and patient follow-up: Koji Takahashi, Toshio Tsuyuguchi, Harutoshi Sugiyama, Masato Nakamura, Junichiro Kumagai, Mutsumi Yamato, Yotaro Iino, Ayako Shingyoji, Hiroshi Ohyama, Shin Yasui, Rintaro Mikata.

Drafting of the manuscript: Koji Takahashi, Toshio Tsuyuguchi, and Yoshihiko Ooka.

\section{ACKNOWLEDGMENTS}

The authors would like to thank all the staff involved in ERCP at the Chiba University Hospital. We also thank Cook Medical for providing the photo of the uncovered self-expandable metal stent and its delivery system. We would also like to thank Enago (www.enago.jp) for the English language review.

\section{CONFLICTS OF INTEREST}

The authors declare no conflicts of interest.

\section{FUNDING}

This research received no specific grant from any funding agency in the public, commercial, or not-for-profit sectors.

\section{REFERENCES}

1. Pavlidis ET, Pavlidis TE. Pathophysiological consequences of obstructive jaundice and perioperative management. Hepatobiliary Pancreat Dis Int. 2018; 17:17-21. https://doi. org/10.1016/j.hbpd.2018.01.008.

2. Wagner HJ, Knyrim K, Vakil N, Klose KJ. Plastic endoprostheses versus metal stents in the palliative treatment of malignant hilar biliary obstruction. A prospective and randomized trial. Endoscopy. 1993; 25:213-8.

3. Mukai T, Yasuda I, Nakashima M, Doi S, Iwashita T, Iwata K, Kato T, Tomita E. Metallic stents are more efficacious than plastic stents in unresectable malignant hilar biliary strictures: a randomized controlled trial. J Hepatobiliary Pancreat Sci. 2013; 20:214-22. https://doi.org/10.1007/ s00534-012-0508-8.

4. Kawakami H, Itoi T, Kuwatani M, Kawakubo K, Kubota Y, Sakamoto N. Technical tips and troubleshooting of endoscopic biliary drainage for unresectable malignant hilar biliary obstruction. J Hepatobiliary Pancreat Sci. 2015; 22:E12-21. https://doi.org/10.1002/jhbp.186. Epub 2014 Nov 7.

5. Isayama H, Hamada $T$, Yasuda $I$, Itoi $T$, Ryozawa $S$, Nakai Y, Kogure H, Koike K. TOKYO criteria 2014 for transpapillary biliary stenting. Dig Endosc. 2015; 27:25964. https://doi.org/10.1111/den.12379. Epub 2014 Dec 4.

6. Freeman ML, Overby C. Selective MRCP and CT-targeted drainage of malignant hilar biliary obstruction with selfexpanding metallic stents. Gastrointest Endosc. 2003; 58:41-9.

7. De Palma GD, Pezzullo A, Rega M, Persico M, Patrone F, Mastantuono L, Persico G. Unilateral placement of metallic stents for malignant hilar obstruction: a prospective study. Gastrointest Endosc. 2003; 58:50-3.

8. Kawamoto H, Tsutsumi K, Harada R, Fujii M, Kato H, Hirao K, Kurihara N, Nakanishi T, Mizuno O, Ishida E, Ogawa T, Fukatsu H, Sakaguchi K. Endoscopic deployment of multiple JOSTENT SelfX is effective and safe in treatment of malignant hilar biliary strictures. Clin Gastroenterol Hepatol. 2008; 6:401-8. https://doi. org/10.1016/j.cgh.2007.12.036. Epub 2008 Mar 7.

9. Park DH, Lee SS, Moon JH, Choi HJ, Cha SW, Kim JH, Seo DW, Lee SK, Park SH, Lee MS, Kim SJ, Kim MH. Newly designed stent for endoscopic bilateral stent-in-stent placement of metallic stents in patients with malignant hilar biliary strictures: multicenter prospective feasibility study (with videos). Gastrointest Endosc. 2009; 69:1357-60. https://doi.org/10.1016/j.gie.2008.12.250.

10. Lee JH, Kang DH, Kim JY, Lee SM, Kim DH, Park CW, Cho HS, Kim GH, Kim TO, Heo J, Song GA, Cho M, Kim $\mathrm{S}$, et al. Endoscopic bilateral metal stent placement for advanced hilar cholangiocarcinoma: a pilot study of a newly designed Y stent. Gastrointest Endosc. 2007; 66:364-9. 
11. Kim JY, Kang DH, Kim HW, Choi CW, Kim ID, Hwang JH, Kim DU, Eum JS, Bae YM. Usefulness of slimmer and open-cell-design stents for endoscopic bilateral stenting and endoscopic revision in patients with hilar cholangiocarcinoma (with video). Clin Gastroenterol Hepatol. 2008; 6:401-8. https://doi.org/10.1016/j. cgh.2007.12.036. Epub 2008 Mar 7.

12. Chahal P, Baron TH. Expandable metal stents for endoscopic bilateral stent-within-stent placement for malignant hilar biliary obstruction. Gastrointest Endosc. 2010; 71:195-9. https://doi.org/10.1016/j.gie.2009.08.006. Epub 2009 Nov 27.

13. Lee TH, Kim TH, Moon JH, Lee SH, Choi HJ, Hwangbo Y, Hyun JJ, Choi JH, Jeong S, Kim JH, Park DH, Han JH, Park $\mathrm{SH}$. Bilateral versus unilateral placement of metal stents for inoperable high-grade malignant hilar biliary strictures: a multicenter, prospective, randomized study (with video). Gastrointest Endosc. 2017; 86:817-827. https://doi. org/10.1016/j.gie.2017.04.037. Epub 2017 May 4.
14. Vienne A, Hobeika E, Gouya H, Lapidus N, Fritsch J, Choury AD, Chryssostalis A, Gaudric M, Pelletier G, Buffet C, Chaussade S, Prat F. Prediction of drainage effectiveness during endoscopic stenting of malignant hilar strictures: the role of liver volume assessment. Gastrointest Endosc. 2009; 70:1109-15. https://doi.org/10.1016/j.gie.2009.05.013. Epub 2009 Jul 31.

15. Takahashi E, Fukasawa M, Sato T, Takano S, Kadokura M, Shindo H, Yokota Y, Enomoto N. Biliary drainage strategy of unresectable malignant hilar strictures by computed tomography volumetry. Gastrointest Endosc. 2010; 71:1959. https://doi.org/10.1016/j.gie.2009.08.006. Epub 2009 Nov 27.

16. Bismuth H, Nakache R, Diamond T. Management strategies in resection for hilar cholangiocarcinoma. Gastrointest Endosc. 2017; 86:817-827. https://doi.org/10.1016/j. gie.2017.04.037. Epub 2017 May 4. 